\title{
Activity, Rotation and Convection in Orion: Are the Data Inconsistent with the MS Activity-Rossby Number Relation?
}

\author{
E. Flaccomio, G. Micela, N. Pizzolato, S. Sciortino \\ INAF - Osservatorio Astronomico di Palermo G.S. Vaiana \\ P. Ventura
}

INAF - Osservatorio Astronomico di Roma

\begin{abstract}
A relation between activity and rotation in young $\sim 1 \mathrm{Myr}$ PMS in often not observed, suggesting that the mechanism responsible for the $\mathrm{X}$ ray emission may differ from the $\alpha-\omega$ dynamo. We re-investigate the matter utilizing recent X-ray and rotational data on the Orion Nebula Cluster (ONC).
\end{abstract}

\section{Introduction}

The origin of activity in $\sim 1$ Myr PMS stars is uncertain. For MS stars activity correlates with the Rossby number, $R_{0}=P_{\text {rot }} / \tau_{\text {conv }}\left(P_{\text {rot }}\right.$ being the stellar rotational period and $\tau_{\text {conv }}$ the convective turnover time $): \log \left(L_{\mathrm{X}} / L_{\mathrm{bol}}\right) \propto \log \left(R_{0}\right)$. This relation (see Pizzolato et al. 2002, PMMSV hereafter) saturates for small $R_{0}$, when $\log \left(L_{\mathrm{X}} / L_{\mathrm{bol}}\right) \sim-3$. Given that for MS stars rotation plays a predominant role, several studies have searched, often with no success, for correlations of PMS activity with rotation alone (e.g. Feigelson et al. 2003). Here we reexamine the matter using our X-ray data on a sample of ONC stars (Flaccomio et al. 2003a), published rotational data (e.g. Herbst et al. 2001), and evolutionary tracks by Ventura et al. (1998, VZMD hereafter).

\section{Data Analysis and Results}

We first estimate convective turnover times for a subset of ONC stars using the VZMD tracks (solar metallicity, MLT) for masses in the 0.6-1.6 $M_{\odot}$ range and optical data from Hillenbrand et al. (1997). According to the models, stars have much larger $\tau_{\text {conv }}$ at the age of the ONC $\left(\sim 10^{6}\right.$ years $)$ respect to MS values. In order to compare the activity of ONC stars with that of MS stars we use the PMMSV sample. Using the X-ray and optical data in PMMSV and the VZMD tracks, we put these stars in the $\log \left(L_{\mathrm{X}} / L_{\text {bol }}\right)$ vs. $\log \left(R_{0}\right)$ plane (Fig. 1).

The $\log \left(L_{\mathrm{X}} / L_{\mathrm{bol}}\right)$ vs. $\log \left(P_{\text {rot }}\right)$ plot for ONC members in the 0.6-1.6 $M_{\odot}$ range (not shown) shows no correlation and the high activity levels of the slowest rotators is incompatible with the MS data. The same lack of correlation appears in the $\log \left(L_{\mathrm{X}} / L_{\text {bol }}\right)$ vs. $\log \left(R_{0}\right)$ plot. However, because of their large $\tau_{\text {conv }}$, all the ONC members have $R_{0}$ values corresponding to saturated MS activity. A 

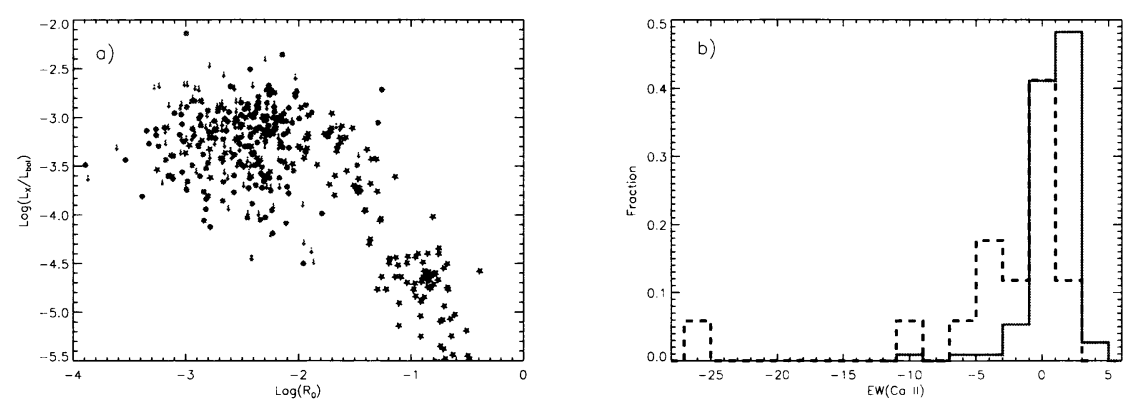

Figure 1. (a) $\log \left(L_{\mathrm{X}} / L_{\text {bol }}\right)$ vs. $\log \left(R_{0}\right)$. Circles and upper-limit arrows: ONC stars; stars: MS sample from PMMSV. (b) Distribution of Ca II line equivalent width for ONC members with $\log \left(L_{\mathrm{X}} / L_{\mathrm{bol}}\right)>-3.4$ (black) and $\log \left(L_{\mathrm{X}} / L_{\text {bol }}\right)<-3.9$ (gray).

correlation with $R_{0}$ (or $P_{\text {rot }}$ ) is thus not expected. Several discrepant stars however appear in such a plot with activity well below the MS locus.

To extend our sample to lower mass stars than accessible with the VZMD tracks, we use the Siess et al. (2001, SDF hereafter) calculations (available down to $0.1 M_{\odot}$ ). SDF do not provide $\tau_{\text {conv }}$, which is needed to compute the Rossby number. However, the VZMD models indicate a tight correlation between $\tau_{\text {conv }}$ and the depth of the convective region (provided by SDF). Aware of the uncertainty of extending this relation to different stellar masses, we use it to estimate $\tau_{\text {conv }}$ and $R_{0}$. Figure 1a shows the $\log \left(L_{\mathrm{X}} / L_{\text {bol }}\right)$ vs. $\log \left(R_{0}\right)$ plot for this enlarged sample. It is similar to that obtained using the VZMD tracks. Almost all ONC stars have estimated $R_{0}$ within the saturated regime of MS stars. A few stars, however, show too low activity for their Rossby number. Following Flaccomio et al. $(2003 \mathrm{a}, \mathrm{b})$ we investigate the possibility that the X-ray activity of these stars is lowered by an accretion disk. Figure $1 \mathrm{~b}$ shows distributions of the $\mathrm{Ca}$ II $(\lambda=8542 \AA)$ equivalent width, an accretion indicator, for two sub-samples of the stars in Fig. 1a segregated by $\log \left(L_{\mathrm{X}} / L_{\text {bol }}\right)$. The two distributions differ at the of $3.7 \sigma$ level (Wilcoxson rank-sum test), confirming that low activity stars are characterized by strong accretion. Hence PMS stars activity has likely the same origin as in MS stars, but it is somehow lowered by accretion disks.

\section{References}

Feigelson, E. D., et al. 2003, ApJ 584, 911

Flaccomio, E., Damiani, F., Micela, G., et al. 2003a , ApJ 582, 398

Flaccomio, E., Micela, G., Sciortino. S., 2003b, A\&A 397, 611

Herbst, W., Bailer-Jones, C.A.L., \& Mundt, R. 2001, ApJ 554, L197

Hillenbrand, L. A. 1997, AJ 113, 1733

Pizzolato, N., Maggio A., Micela, G., Sciortino, S., Ventura, P., 2003, A\&A 397, 147

Siess, L., Dufour, E., \& Forestini, M. 2000, A\&A 358, 593

Ventura, P., Zeppieri, A., Mazzitelli, I., \& D'Antona, F. 1998, A\&A 334, 953 